\title{
Mood Disorders among Chronic Rhino-Sinusitis Patients
}

\section{Nasim Gharaati ${ }^{1}$, Ehssan Amini ${ }^{1}$, Mehran Abdollahi' ${ }^{1}$, Mohammad R Maracy², Sayyed Hamidreza Abtahi ${ }^{3}$ and Gholam R Kheirabadi**}

${ }^{1}$ Medical Students' Research Center, Isfahan University of Medical Sciences, Isfahan, Iran

${ }^{2}$ Department of Community Medicine, School of Medicine, Isfahan University of Medical Sciences, Hezar Jerib Avenue, Isfahan, Iran

${ }^{3}$ Department of Otolaryngology, School of Medicine, Isfahan University of Medical Sciences, Isfahan, Iran

${ }^{4}$ Behavioral Sciences Research Centre, Department of Psychiatry, School of Medicine, Isfahan University of Medical Sciences, Isfahan, Iran

\section{Abstract}

Background: Chronic rhinosinusitis (CRS) is one of the prevalent inflammatory diseases all over the world. Recent studies have shown some kind of relation between CRS and mood disorders such as depression and anxiety this study aims to assess this relationship in an outpatient setting.

Methods: In this case control study 162 CRS patients and 151 healthy subjects as control group were selected. Hospital anxiety and depression scale (HADS) questionnaire was presented to all of them. Depression and anxiety subscales of the questionnaire were compared in two groups.

Results: According to depression $21.6 \%$ of case group and $21.2 \%$ of control group were scaled as depressed and $34 \%$ of cases and $32.7 \%$ of control subjects were in need of medical care for anxiety. None of these differences were statistically significant.

Conclusion: Although depression and anxiety are prevalent in CRS patients but it is not more prevalent than normal population

Keywords: Chronic rhino-sinusitis; Mood disorders; Depression; Anxiety

\section{Introduction}

Chronic rhinosinusitis (CRS) is one of the most prevalent inflammatory diseases defined as inflammation of nasal cavity and paranasal sinuses lasting at least 12 consecutive weeks, presenting with symptoms such as itching, sneezing, rhinorrhea, and nasal congestion [1]. Its prevalence among US adult population was estimated about $16 \%$ according to 2010 national health interview survey [2]. In studies conducted in Iran the estimated prevalence of CRS is about $14.5 \%$ to $22.5 \%$ in adult population $[3,4]$. Regarding high prevalence of CRS it's rational to expect a high socioeconomic burden for this disorder as it was shown in Bhattacharyya study, who reported the economic burdens of CRS patients to be $\$ 8.6$ billion annually [5]. Looking at CRS as a chronic disease, lots of studies sought its effect on quality of life. Most of these studies reported a significantly impaired health related quality of life in these patients [6]. Searching for the reasons of diminished quality of life in these patients and the fact that psychological disorders usually have comorbidity with chronic diseases, has navigated researches attention toward the association of CRS and psychological problems especially depression and anxiety. Some of these studies reported a very high prevalence of depression and anxiety in CRS patients. Brandsted et al. reported the prevalence of depression to be $26 \%$ among patients with sinonasal symptoms [7]. In another study using hospital anxiety and depression scale (HADS), $25.9 \%$ and $14.7 \%$ of CRS patients had high levels of anxiety and depression, respectively [8]. In a cohort study in 2011 the incidence of depression in patients with CRS was 77\% more than control group with a hazard ratio of 1.56 [8] while these studies report depression as a highly prevalent disorder in CRS patients there are some other studies which report the prevalence of highly probable depression as low as 3 to $4 \%$ [9,10]. In 2016 Schloser et al. reviewed 13 studies and reported the prevalence of probable depression to be from 11 to $40 \%$ [11]. Considering this wide range of results and the fact that causality in this association is not yet understood, it seems necessary to conduct more studies in this field. The aim of this study was to determine the average score and the prevalence of depression and anxiety within CRS patients using HADS questionnaire.

\section{Methods}

In present study we used a case-control design to determine the state of anxiety and depression in CRS patients in comparison with non CRS subjects, using a HADS questionnaire. The study was held in the otolaryngology outpatient clinic of Alzahra hospital (which is a tertiary care center affiliated to Medical university of Isfahan, Iran), from November 2015 to July 2016. CRS patients were diagnosed by an expert otolaryngologist based on European adult Sinusitis guideline [12], but due to economic limitations radiographic assessment and endoscopic evaluation was not administered. As both genetic and environmental features play a significant role in psychological disorders the control group was selected among patient's attendants in order to minimize sociocultural and genetic differences. Subjects in the control group were examined by the otolaryngologist to rule out any chance of rhinosinusal disorders. Subjects less than 18 years old and those with a history of psychiatric disorders other than depression and anxiety, surgical operation or hospitalization in previous month, and the ones who were candidate for surgical operation in following month were not included. The questionnaire was provided to the patients and control subjects in the same time and same room. Considering different levels of patient's socioeconomic status, one of the research team members was present as the patient filled the questionnaire in order to explain the study to him/her and answer their questions in case of need.

\section{Questionnaire}

The questionnaire we used consists of three main parts. In the first part demographic variables including date of birth, gender (male/

*Corresponding author: Gholam Reza Kheirabadi, Associate Professor of Psychiatry, Behavioral Sciences Research Centre, Department of Psychiatry, School of Medicine, Isfahan University of Medical Sciences, Isfahan, Iran, Tel: +989133174349; E-mail: kheirabadi@bsrc.mui.ac.ir

Received: June 15, 2017; Accepted: June 27, 2017; Published: July 04, 2017

Citation: Gharaati N, Amini E, Abdollahi M, Maracy MR, Abtahi SH, et al. (2017) Mood Disorders among Chronic Rhino-Sinusitis Patients. J Psychiatry 20: 427 doi:10.4182/2378-5756.1000427

Copyright: (c) 2017 Gharaati N, et al. This is an open-access article distributed under the terms of the Creative Commons Attribution License, which permits unrestricted use, distribution, and reproduction in any medium, provided the original author and source are credited 
female), educational level (illiterate or primary education/high school education/academic education), marital status (single/divorced/ married/widowed), occupational status (employed/unemployed/ student/retired) were asked. Second part consisted of 4 questions, 2 of them about patient's history of his/ her past medical and drug history, one question about severity of CRS in relation to different seasons and a question about CRS duration. The third section was HADS questionnaire which is designed by Zigmond and Snaith in 1982 [13]. This questionnaire has been developed to assess depression and anxiety scales in the setting of a medical hospital outpatient clinic. It consists of 14 multiple choices questions that the patient should answer to base on his/her last week experiences. Seven questions indicate depression and anxieties. Each question can be rated from 0 to 3 based on the patient's choice so the each subscale can be rated from 0 to 21 . The score from 0 to 7 indicates no anxiety or depression, 8-11 indicates probable or mild anxiety or depression and the score more than 11 is translated as highly probable depression or anxiety which need medical care. In this study we used Persian format of the questionnaire which was developed by Montazeri et al. with a Cronbach's alpha coefficient of 0.78 for anxiety and 0.86 for depression subscale [14].

Statistical analysis Sample size was calculated using compare of means formula, with an $80 \%$ power and $5 \%$ significance, resulted in 150 case-control sets (one control for each case) were needed.

Data were analyzed, using SPSS version 19.0 (Statistical Package for the Social Sciences). Student T-test and chi square were used to assess the differences of quantitative and categorical variables between two groups. Logistic regression model was used to assess the odds of CRS occurrence in relation to age, gender, education, marital status, disease history, drug history, depression, and anxiety. Values are presented in mean and standard deviation (SD), percentage, odds ratio (OR) and confidence interval (CI). Differences with a p value of less than 0.05 were considered as statistically significant. Missing values of each group were replaced by mode and mean of the same group for categorical and quantitative variables, respectively.

\section{Results}

One hundred and sixty two CRS patients ( $45.7 \%$ male) with the mean age of $35.3 \pm 11.4$ and 151 control subjects ( $45.0 \%$ male) with the mean age of $33.5 \pm 8.5$ were included in this study. Demographic factors distribution differences between case and control subjects were not statistically significant (Table 1). We could not gather control match for 11 cases because 8 patients had visited the physician by themselves, 2 of the patient's attendants were diagnosed as having kind of rhinosinusal disorder and 1 of them was less than 18 years old. Forty two (25.9\%) patients had a history of some kind of diseases other than CRS and 31 (19.1\%) of them used medication, whereas in control group 26 (17.9\%) had a positive medical history and $18(11.9 \%)$ reported medication use but this difference was not meaningful $(\mathrm{P}>0.05)$ (Table 1$)$.

Mean depression score was $7.2 \pm 4.2$ among CRS patients and $7.2 \pm 4.3$ in control group and the difference was not statistically significant ( $\mathrm{P}$ value $=0.893$ ). Using HADS scoring system $86(53.1 \%)$ CRS patients were categorized as normal, 41(25.3\%) was scored as having mild depression, and 35(21.6\%) of them were depressed and in need of medical care, the distribution in control group was $71(47.0 \%)$, $48(31.8 \%)$ and $32(21.2 \%)$ respectively and the difference between two groups was not significant $(\mathrm{P}$ value $=0.420)$.

Mean anxiety score in case and control groups was $9.15 \pm 4.38$ and $9.07 \pm 4.39$ respectively and did not differ statistically ( $\mathrm{p}$-value $=0.870$ ). Thirty four percent of CRS patients and $32.7 \%$ of control subjects were diagnosed as in need of medical care for anxiety and the difference between two groups was not statistically significant $(\mathrm{P}$ value $=0.971$ ). Data on depression and anxiety scores and scale in each group is presented in Table 1 .

\begin{tabular}{|c|c|c|c|c|}
\hline \multicolumn{2}{|c|}{ Characteristics } & \multirow{2}{*}{$\begin{array}{c}\text { CRS patients } \\
35.3 \pm 11.4\end{array}$} & \multirow{2}{*}{$\begin{array}{c}\text { Control } \\
33.5 \pm 8.5\end{array}$} & \multirow{2}{*}{$\begin{array}{c}\text { P-value* }^{*} \\
0.106\end{array}$} \\
\hline Age: Year & Mean \pm SD & & & \\
\hline \multirow{2}{*}{ Sex: $\mathbf{N}(\%)$} & Male & $74(45.7 \%)$ & $68(45.0 \%)$ & \multirow{2}{*}{0.909} \\
\hline & Female & $88(54.3 \%)$ & $83(55.0 \%)$ & \\
\hline \multirow{3}{*}{ Education: N (\%) } & Illiterate/primary & $35(21.6 \%)$ & $26(17.2 \%)$ & \multirow{3}{*}{0.327} \\
\hline & High school & $70(43.2 \%)$ & $60(39.7 \%)$ & \\
\hline & Academic & $57(35.2 \%)$ & $65(43.0 \%)$ & \\
\hline \multirow{4}{*}{ Occupation: N (\%) } & Employed & $76(46.9 \%)$ & $73(48.3 \%)$ & \multirow{4}{*}{0.816} \\
\hline & Student & $25(15.4 \%)$ & $23(15.2 \%)$ & \\
\hline & Retired & $9(5.6 \%)$ & $5(3.3 \%)$ & \\
\hline & Unemployed & $52(32.1 \%)$ & $50(33.1 \%)$ & \\
\hline \multirow{2}{*}{ Marital status: $\mathbf{N}(\%)$} & Single/divorced & $48(29.6 \%)$ & $46(30.5 \%)$ & \multirow{2}{*}{0.872} \\
\hline & Married/widowed & $114(70.4 \%)$ & $105(69.5 \%)$ & \\
\hline \multirow{2}{*}{ Positive medical history: $\mathrm{N}(\%)$} & Yes & $42(25.9 \%)$ & $26(17.2 \%)$ & \multirow{2}{*}{0.062} \\
\hline & No & $120(74.1 \%)$ & $125(82.8 \%)$ & \\
\hline \multirow{2}{*}{ Medication history: N (\%) } & Yes & $31(19.1 \%)$ & $18(11.9 \%)$ & \multirow{2}{*}{0.079} \\
\hline & No & $131(80.9 \%)$ & $133(88.1 \%)$ & \\
\hline Depression score & Mean \pm SD & $7.16 \pm 4.16$ & $7.23 \pm 4.35$ & 0.893 \\
\hline \multirow{3}{*}{ Depression scale: $\mathbf{N}(\%)$} & Normal & $86(53.1 \%)$ & $71(47.0 \%)$ & \multirow{3}{*}{0.420} \\
\hline & Mild & $41(25.3 \%)$ & $48(31.8 \%)$ & \\
\hline & Indicating medical care & $35(21.6 \%)$ & $32(21.2 \%)$ & \\
\hline Anxiety score & Mean \pm SD & $9.15 \pm 4.38$ & $9.07 \pm 4.39$ & 0.870 \\
\hline \multirow{3}{*}{ Anxiety scale: N(\%) } & Normal & $59(36.4 \%)$ & $56(37.3 \%)$ & \multirow{3}{*}{0.971} \\
\hline & Mild & $48(29.6 \%)$ & $45(30.0 \%)$ & \\
\hline & Indicating medical care & $55(34.0 \%)$ & $49(32.7 \%)$ & \\
\hline
\end{tabular}

Table 1: Comparison of demographic and clinic characteristics based on the allergic group and controls. 
A logistic regression was performed to ascertain the effects of age, gender, education, marital status, disease history, drug history, depression, and anxiety on CRS occurrence. None of the variables had a statistically significant odds ratio (Table 2 ).

\section{Discussion and Conclusion}

It was hypothesized that depression and anxiety are highly prevalent in CRS patients in comparison to healthy population. In this study it was proved that these psychological disorders are prevalent among CRS patients but when it comes to comparison with normal healthy population the hypothesis failed and there was no significant difference.

We estimated the prevalence of depression to be about $21 \%$ in CRS patients which is in line with other studies that used HADS questionnaire and reported the prevalence of $11-23.8 \%$ [11]. The main reason of this wide range in these studies can be related to different HADS cut-off points used by them. Where the studies which used score 6 as their cut-off point for depression diagnosis reported the highest prevalence and the study which used 11 as cut-off reported the lowest $[8,15]$. In our study we used 11 as depression cut-off score but the prevalence of depression was like the ones who used lower scores to define depression and it shows a higher prevalence of depression in our study. In other studies which used different methods to evaluate depression, the results are somehow different. Jung et al. reported the prevalence of mild and moderate depression to be about $40 \%$ using Beck depression inventory (BDI) scale $[9,16]$. Schlosser et al. used BDI too and reported the prevalence of $31 \%$ in CRS patients [17]. One important clue which can explain the differences in depression prevalence report of our study in contrast to studies which used BDI is that HADS cannot assess somatic symptoms of depression as exhaustion or sleep disturbances whereas BDI assess both somatic and mood symptoms. So, considering CRS as a chronic disease which impairs sleep and can cause day time exhaustion; the difference seems logical.

Thirty four percent of CRS patients were in need of medical care according to their anxiety score. This is in line with a study conducted in 2013 by Nanayakkara et al. that reported the prevalence of $32 \%$ in 57 CRS patients in London [15]. Other studies using HADS questionnaire reported lower prevalence in a range of $12 \%$ to $26 \%[8,10,18]$. Herein different studies used different scoring systems, too. And this can be the reason of different prevalence. Also, it should be considered that in some studies subjects were selected through patients undergoing a sinonasal surgery and it can make them stressful.

Comparing psychological disorders between CRS patients and control group no significant difference was seen and this is in contrast with most studies in this field. Katotomichelakis et al. in a study evaluated psychological status among CRS patients, observed that olfactory impairment can play a role in promoting psychological disorders. But when it comes to patients with normal olfactory function psychological assessments reveal no impairment [19]. In another study Tomum et al. showed that HADS score and rhinosinusal disability index are highly correlated but there were no correlation with LudKennedy endoscopic score [18]. Considering these two studies it is possible that dysfunctions caused by CRS are in relation with more psychological problems. Unfortunately we didn't assess CRS-caused dysfunctions in our study to have a better estimate of differences between the two groups.

Schlosser et al. in a study in 2016 used BDI as depression scale in a case control study. It was shown that CRS patients had depression twice more than control subjects but depression prevalence diagnosed by a clinician did not differ between two groups [17]. As mentioned before BDI assess both somatic and cognitive symptoms as HADS assess just cognitive ones and it is interesting that in Schlosser study BDI somatic subscale scores were significantly different but cognitive differences were not statistically significant. And this finding is in line with our study and it can be hypothesized that CRS effects on mood is through somatic impairments and not cognitive ones.

In some studies using functional magnetic resonance imaging (fMRI) some neural pathways in insula and anterior singulate cortex has been found that process both pain and mood [20]. And also in depressed and anxious subjects a hypothalamic-pituitary-adrenal axis is activated that causes cytokine response resulting in a chronic preinflammatory state $[21,22]$. Therefore it is possible that CRS causes mood disturbances through somatic symptoms at first and then this mood disorder causes more severe CRS symptoms and this continues in a cyclic manner. Considering this hypothesis it seems rational that depression spectrum disorders incidence rise in CRS subjects during

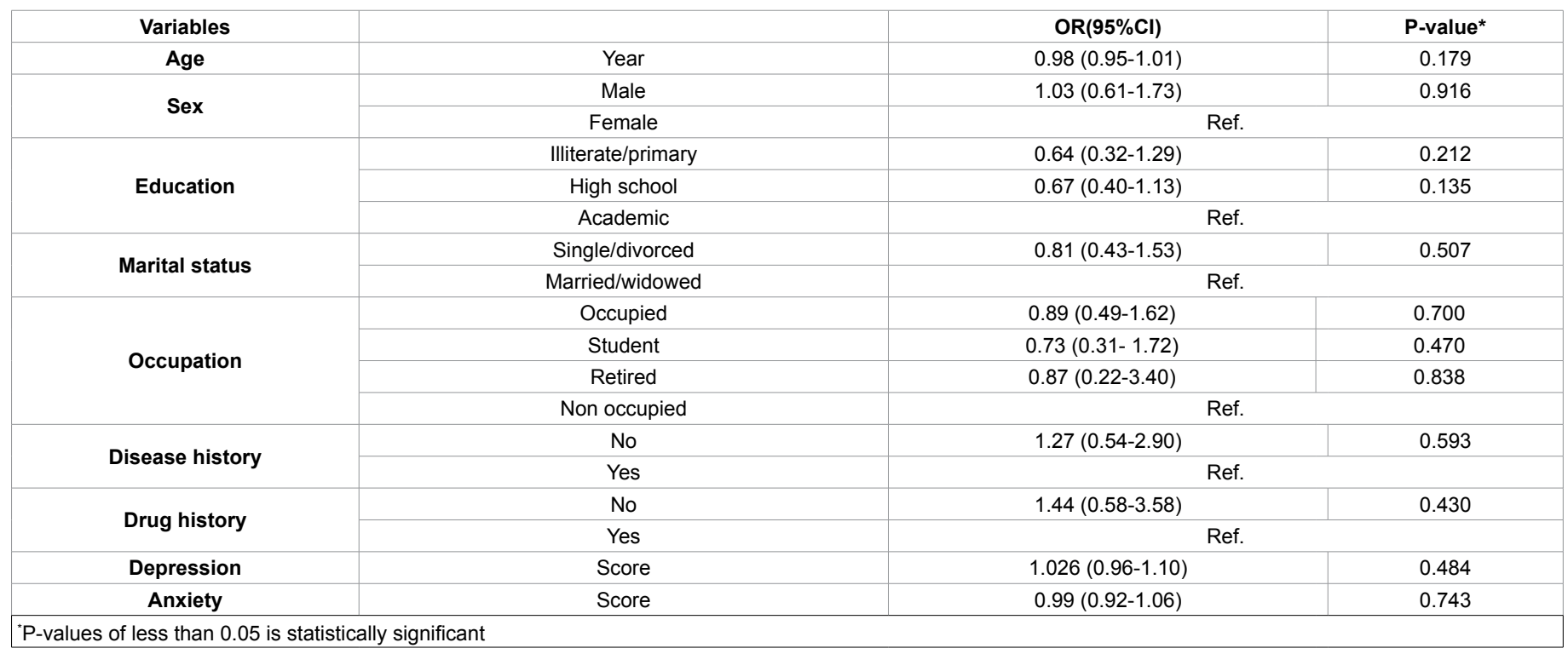

Table 2: Association of demographic and clinic characteristics on allergic groups using logistic regression model. 
a period of time as it was shown in a cohort study in Taiwan on 15,371 CRS cases and 61,484 non-CRS controls, where depression incidence in cases was approximately two times more than control group [23].

In this study CRS diagnosis was based on history and physical examination alone, and due to economical limitations we didn't use radiologic and endoscopic assessments so we couldn't categorized CRS patients based on the severity, we didn't consider functional impairment in our study, either. These are the most important limitations of our study. Another limitation of this study is that our control group was not a good representative of normal healthy population. We tried to control genetic and socioeconomic factors by selecting patients attendants (most of them their siblings), but patient's disease can cause adverse effects on their attendant's mood, too.

As genetic plays an important role in mood disorders it is suggested that future studies consider this factor as one of the confounder variables. Also studies has defined the role of some specific neurotransmitters such as serotonin and dopamine in both mood disorders and inflammatory processes [24]; focusing on these subjects in future studies can illuminate the way through association of these disorders.

\section{Acknowledgements}

The Authors declare that there is no conflict of interest. We sincerely acknowledge all subjects, who have accepted to participate in this study and shared their innate feelings with us, kindly.

\section{References}

1. Benninger M, Ferguson B, Hadley J, Hamilos D, Jacobs M, et al. (2003) Adult chronic rhinosinusitis: Definitions, diagnosis, epidemiology, and pathophysiology. Otolaryngol Head Neck Surg 129: S1-S32.

2. Pleis JR, Lucas JW (2009) Summary health statistics for U.S. adults: Nationa Health Interview Survey. Vital Health Stat 240: 1-159.

3. Mohammadzadeh I, Ghafari J, Savadkoohi RB, Tamaddoni A, Dooki MRE, et al. (2008) The prevalence of asthma, allergic rhinitis and eczema in north of Iran. Iran J Pediatr 18: 117-122.

4. Varasteh AR, Fereidouni M, Shakeri MT, Vahedi F, Abolhasani A, et al. (2009) Prevalence of allergic disorders among the population in the city of Mashhad Northeast Iran. J Public Health 17: 107-112.

5. Bhattacharyya N (2011) Incremental health care utilization and expenditures for chronic rhinosinusitis in the United States. Ann Otol Rhinol Laryngol 120: 423-427.

6. Rudmik L, Smith TL (2011) Quality of life in patients with chronic rhinosinusitis. Curr Allergy Asthma Rep 11: 247-252.

7. Brandsted R, Sindwani R (2007) Impact of depression on disease-specific symptoms and quality of life in patients with chronic rhinosinusitis. Am J Rhinol 21: $50-54$.
8. Wasan A, Fernandez E, Jamison RN, Bhattacharyya N (2007) Association of anxiety and depression with reported disease severity in patients undergoing evaluation for chronic rhinosinusitis. Annals of Otology, Rhinology \& Laryngology 116: 491-497.

9. Jung YG, Lee JS, Park GC (2014) Does post-infectious olfactory loss affect mood more severely than chronic sinusitis with olfactory loss? Laryngoscope 124: $2456-2460$

10. Sahlstrand-Johnson P, Ohlsson B, Von Buchwald C, Jannert M, Ahlner-Elmqvist $M$ (2011) A multi-centre study on quality of life and absenteeism in patients with CRS referred for endoscopic surgery. Rhinology 49: 420-428.

11. Schlosser RJ, Gage SE, Kohli P, Soler ZM (2016) Burden of illness: A systematic review of depression in chronic rhinosinusitis. Am J Rhinol Allergy 30: 250-256.

12. Rosenfeld RM, Piccirillo JF, Chandrasekhar SS, Brook I, Kumar KA, et al (2015) Clinical practice guideline (update) adult sinusitis. Otolaryngol Head Neck Surg 152: S1-S39.

13. Zigmond AS, Snaith RP (1983) The hospital anxiety and depression scale. Acta psychiatrica scandinavica 67: 361-370.

14. Montazeri A, Vahdaninia M, Ebrahimi M, Jarvandi S (2003) The Hospita Anxiety and Depression Scale (HADS): Translation and validation study of the Iranian version. Health Qual Life Outcomes 1: 1

15. Nanayakkara JP, Igwe C, Roberts D, Hopkins C (2013) The impact of mental health on chronic rhinosinusitis symptom scores. Eur Arch Otorhinolaryngol 270: 1361-1364

16. Beck AT, Steer RA, Brown GK (1996) Manual for the beck depression inventoryII. Psychological Corporation, San Antonio, TX.

17. Schlosser RJ, Storck K, Cortese BM, Unde TW, Rudmik L, et al. (2016) Depression in chronic rhinosinusitis: A controlled cohort study. Am J Rhino Allergy 30: 128-133.

18. Tomoum MO, Klattcromwell C, DelSignore A, Ebert C, Senior BA (2015) Depression and anxiety in chronic rhinosinusitis. Int Forum Allergy Rhinol 5 674-681.

19. Katotomichelakis M, Simopoulos E, Tzikos A, Balatsouras D, Tripsianis G, et al. (2014) Demographic correlates of anxiety and depression symptoms in chronic sinonasal diseases. Int J Psychiatry Med 48: 83-94.

20. Zubieta JK, Ketter TA, Bueller JA, Xu Y, Kilbourn MR, et al. (2003) Regulation of human affective responses by anterior cingulate and limbic mu-opioid neurotransmission. Arch Gen Psychiatry 60: 1145-1153.

21. Ploghaus A, Tracey I, Gati JS, Clare S, Menon RS, et al. (1999) Dissociating pain from its anticipation in the human brain. Science 284: 1979-1981.

22. Raison CL, Capuron L, Miller AH (2006) Cytokines sing the blues: Inflammation and the pathogenesis of depression. Trends Immunol 27: 24-31.

23. Hsu CL, Wang TC, Shen TC, Huang YJ, Lin CL, et al. (2016) Risk of depression in patients with chronic rhinosinusitis: A nationwide population-based retrospective cohort study. J Affect Disord 206: 294-299.

24. Ciprandi G, De Amici M, Tosca M, Alesina R, Marseglia G, et al. (2011) Serotonin in allergic rhinitis: a possible role for behavioural symptoms. Iran J Allergy Asthma Immunol 10: 183-188. 